\title{
Topic Enhanced Sentiment Spreading Model in Social Networks Considering User Interest
}

\author{
Xiaobao Wang, ${ }^{1}$ Di Jin, ${ }^{1, *}$ Katarzyna Musial, ${ }^{2}$ Jianwu Dang ${ }^{1,3}$ \\ ${ }^{1}$ College of Intelligence and Computing, Tianjin University, Tianjin 300350, China, \\ ${ }^{2}$ Advanced Analytics Institute, School of Software, University of Technology Sydney, Australia, \\ ${ }^{3}$ School of Information Science, Japan Advanced Institute of Science and Technology, Japan \\ \{wxbxmt, jindi\}@tju.edu.cn, katarzyna.musial-gabrys@uts.edu.au, jdang@jaist.ac.jp
}

\begin{abstract}
Emotion is a complex emotional state, which can affect our physiology and psychology and lead to behavior changes. The spreading process of emotions in the text-based social networks is referred to as sentiment spreading. In this paper, we study an interesting problem of sentiment spreading in social networks. In particular, by employing a text-based social network (Twitter), we try to unveil the correlation between users' sentimental statuses and topic distributions embedded in the tweets, then to automatically learn the influence strength between linked users. Furthermore, we introduce user interest to refine the influence strength. We develop a unified probabilistic framework to formalize the problem into a topic-enhanced sentiment spreading model. The model can predict users' sentimental statuses based on their historical emotional status, topic distributions in tweets and social structures. Experiments on the Twitter dataset show that the proposed model significantly outperforms several alternative methods in predicting users' sentimental status. We also discover an intriguing phenomenon that positive and negative sentiment is more relevant to user interest than neutral ones. Our method offers a new opportunity to understand the underlying mechanism of sentimental spreading in online social networks.
\end{abstract}

\section{Introduction}

With the transformation in Web usage, from information production to information consumption and sharing, numerous social media services, e.g., Facebook and Twitter, have emerged. More and more people are willing to express opinions of events or hot topics. The opinions contain subjects and sentiments, which could be spread by social networks (Guillory et al. 2011). (Kramer, Guillory, and Hancock 2014) found that emotional status can be transferred to others via emotion contagion through a massive experiment on Facebook. (Yang et al. 2016) also found that emotional contagion occurs in image social networks. The contagion process of emotions in text-based social networks is referred to as sentiment spreading (Wang et al. 2017).

\footnotetext{
${ }^{*}$ Corresponding author Copyright (C) 2020, Association for the Advancement of Artificial Intelligence (www.aaai.org). All rights reserved.
}

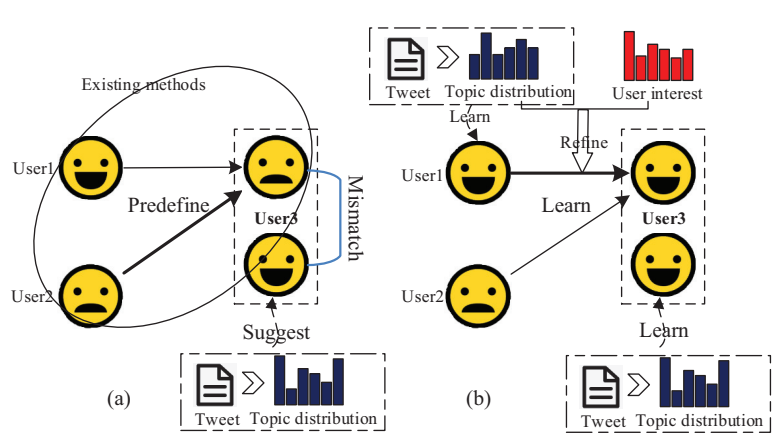

Figure 1: Example scenarios of topic enhanced sentiment spreading. The arrows indicate the direction of sentiment spreading and strength. (a) indicates the situation when adding the topic enhanced mechanism directly into existing methods, and there exists a disparity between the information of network topologies and topic distributions of user-generated content; (b) is our method that automatically balance the effect of these two information and learn the strength, refined by the correlation between user3's interest and topic distribution of tweets posted by neighbors.

Sentiment spreading is a process in which a person or group influences the sentiment or behavior of another person or group, which has attracted considerable research effort. Information diffusion (Wang et al. 2014; 2015) offers a basic condition for sentiment spreading. Several methods based on information diffusion are proposed to study the process of sentiment spreading. For example, (Zhao et al. 2014) proposed a model for sentiment contagion to spread two sentiments: positive and negative. (Wang et al. 2017) categorized information cascades into fine-grained classes and proposed an emotion-based independent cascade model to study the process of sentiment spreading. (Xiong et al. 2018) also proposed an emotional independent cascade model while considering the effect of heterogeneous social networks. These methods use exclusively the information of network topologies (e.g., in-degree and out-degree) or user behaviors (e.g., retweeting and mention), to predefine the influence strength between linked users. 
However, rich information beyond network topologies and user behaviors is embedded in the user-contributed contents (e.g., tweets) in the social network. Specifically, users always post tweets to share their opinions, which can be leveraged to help infer users' sentimental status when studying the process of sentiment spreading. Nevertheless, compared with normal formal text, tweets are much shorter and noisier (Zou, Yang, and Zhang 2018), which may destroy the quality of predicted results. Fortunately, tweets always relate to specific topics or events (He et al. 2017; Jin et al. 2018), while different topics or events have different sentimental tendencies (Ahuja, Wei, and Carley 2017), e.g., political and disasters have more negative sentiment than positive ones, while entertainment is reverse. Thus, considering topic distribution embedded in tweet (i.e., topic enhanced mechanism) may be able to help infer users' sentimental status more accurately when studying the evolution of sentimental spreading.

Even so, it is challenging for existing methods to consider such topic enhanced mechanism directly. Since there may exist a disparity between the information of network topologies and topic distributions of user-generated content. Consider an example scenario in Twitter shown in Fig.1(a). Based on the influence strength between user3 and his neighbors (i.e., user1 and user2) defined by network topology directly, existing methods would suggest that user3 is more likely to be in negative sentiment. When considering the topic distribution of the tweet posted by user3, this user is suggested to be in positive sentiment, which contradicts the results obtained based on the network topology. Thus, there is a need to propose a new sentiment spreading model in order to be able to automatically balance the effect of these two information to alleviate the mismatch brought in Fig. 1(a), when integrating the topic enhanced mechanism as shown in Fig. 1(b). However, users are not likely to post tweets all the time, so considering the topic enhanced mechanism will lead to the situation where some users do not have the information of tweets at some times. Thus, the prediction results of these users may be still not accurate enough.

Fortunately, user interest (i.e., concerned topics), as an attribute of human beings, is relatively stable and is a key factor in the process of information diffusion (Lagnier et al. 2013; Varshney, Kumar, and Gupta 2014; Hao et al. 2016). The higher a user's interest in a certain topic, the more easily a tweet on that topic will be disseminated by that user. Thus, user interest can be used to refine the influence strength between users, even when users do not post tweets all the time. As shown in Fig. 1(b), the correlation between user3's interest and topic distribution of the tweet posted by user1 and user2 can be used to refine the influence strength, thus to alleviate the deficiency of information of tweets.

In this paper, we propose the new approach using topic distributions embedded in tweets to enhance traditional sentiment spreading based purely on topology, meanwhile considering user interests and aim to infer the dynamics of users' sentiment status in a given online social network. Specifically, we propose an effective unified probabilistic graphical model based on factor graph, topic-enhanced sentiment spreading model (TSSM), which uncovers the cor- relation between topic distribution embedded in tweet and user sentimental status; in the meantime, automatically balances the effect of the information of network topology and tweets, and learns the spreading strength between each pair of neighbors integrating above correlation; and finally, integrates user interest into the model to refine the spreading strength.

We summarize our technical contributions as follows:

- We design three kinds of factor functions in a unified probabilistic graphical model to capture underlying mechanism of how tweets' topic distributions help infer sentimental status, then to automatically balances the effect of the information of network topology and tweets, and learn the influence strength considering users' interest and make our unified model describe the sentiment spreading more precisely.

- We developed an efficient inference algorithm based on Loopy Belief Propagation to infer parameters and unknown labels.

- We conduct extensive experiments to validate the proposed model over several baselines. Experimental results show that the proposed model achieves improvement over other approaches.

The rest of the paper is organized as follows. We define the problem we try to solve in Section 2, introduce the proposed model and the basic inference algorithm in Section 3, and present experimental results in Section 4. We conclude with some discussions in Section 5.

\section{Problem Definition}

We are given a text-based social network (e.g., Twitter) that represents relationships between users, where users can post tweets. We formally define the set of tweets as $W$. For each content item $w \in W$, we have the user $v$ who has posted $w$, the timestamp $t_{w}$ of $w$, and a $K$ dimensional vector $x_{w}=$ $\left\{x_{w 1}, \ldots, x_{w K}\right\}$, where $x_{w k}$ donates the probability that the tweet $w$ belongs to topic $k$.

More precisely, we incorporate tweets and social network information in a text-based social network to study sentiment spreading.

A text-based social network is a directed graph $G=$ $(V, W, E, L)$. There are two vertex sets: a set of users (i.e. $V)$ and a set of tweets (i.e. $W$ ). Edges in $E$ represent relationships between users $\{(u, v) \mid u \in V, v \in V\}$, indicating that $u$ follows $v$, and user-tweet relations $\{(v, w) \mid v \in V, w \in$ $W\}$, indicating that user $v$ posts $w$. $L$ denotes users' interests, where $l_{v}$ is the interest of user $v$.

In this work, we aim to study sentiment spreading in a given microblogging social network $G$. We use a $|V| \times T$ matrix Y to denote users' sentimental status, where $y_{v t}$ represents $v^{\prime} s$ sentiment at time $t(t \leq T)$. Due to the sparsity and noisy of contents in microblogging social network, we consider three sentimental status: \{negative, neutral, positive $\}$. We define the prediction task addressed in this paper as below:

Give a text-based social network $G$, a specific time $t$ and sentimental status of users within time $[1, t-1]$, our goal is to learn a function 


$$
\chi=(V, W, E, L), T, Y_{1 \ldots t-1} \rightarrow Y_{t}
$$

\subsection{Model Description}

\section{Proposed Model}

We proposed a novel unified model, topic-enhanced sentiment spreading model (i.e. TSSM), to describe the sentiment spreading in a given social network, which integrates into topic enhanced mechanism and considers users' interests, thus to automatically learn the influence strength between users and predict the sentimental status of users in the future. According to our discussion, the topic distribution of tweet posted by user $v$ at time $t$ is able to express his current sentimental status. Besides, the sentimental status of the user $v$ at time $t$, i.e. $y_{v t}$, will be influenced by the sentimental status of her friends at $t-1$,and refined by the extent that user $v$ is interested in the tweets posted by her friends at $t-1$. Finally, $y_{v t}$ is also influenced by their past sentiment status, which represents the continuation of one's personal sentiment state and has been proven to exist (Yang et al. 2016; Wu et al. 2017; Cai, Jia, and Han 2018).

In general, the main intuition of our model is to 1) learn the correlation between topic distributions embedded in tweet and user sentimental status; 2) learn the influence strength between users by considering users' interests; and (3) learn the dependency between sentimental status of the same user at adjacent time stamps in a unified framework. We then will be able to predict the sentimental status of users.

Based on the above ideas, we propose a partially-labeled factor graph model to learn the function . Factor graph factorizes the "global" probability as a product of "local" factor functions, each of which depends on a subset of variables in the graph (Kschischang et al. 2001). In the proposed model, corresponding to the three intuitions, we define the following three factors.

$\sqrt{ }$ Topic factor: $\ell\left(y_{v t}, x_{v t}\right)$ represents the correlation between user $v$ 's sentimental status at time t (i.e., $y_{v t}$ ) and the topic distribution of the content she posts at the same time (i.e., $x_{v t}$ ).

$\sqrt{ }$ Interest-based spreading factor: $\xi\left(y_{v t}, y_{u t-1}, x_{u t-1}, l_{v}\right)$ represents how user $v$ 's sentimental status at time $t$ is influenced by her friend $u$ 's sentimental status at time $t-1$, which is refined by user $v$ 's interest and also can be seem as influence strength between $v$ and $u$.

$\sqrt{ }$ Self-spreading factor: $\partial\left(y_{v t}, y_{v t-\Delta t}\right)$ represents the correlation between user $v$ 's sentimental status at time $t$ and time $t-\Delta t$. It aims to model how one's sentimental status changes over time.

Fig. 2 shows the graphical representation of the TSSM. The observed data contains three types: users' interests, user relationships and tweets posted by users. We extract topic distributions of tweets and users' interests using existing technologies (more details are given in Experiment part). Then we represent the topic distributions as $x$ and users' interests as $L$. We represent user $v_{1}^{\prime} s$ sentimental status at time 1 as $y_{1 t_{1}}$.
We then formally define each factor. We first introduce the topic factor $\ell\left(y_{v t}, x_{v t}\right)$. The intuition behind this factor is that topics have different sentimental tendencies and are able to express one's sentiment as shown in Fig. 2. Specifically, given a tweet's topic distribution vector $x_{v t}$, we define $\ell\left(y_{v t}, x_{v t}\right)$ as

$$
\ell\left(y_{v t}, x_{v t}\right)=\frac{1}{Z_{\alpha}} \exp \left\{\alpha_{y_{v t}} x_{v t}\right\}
$$

where $\alpha_{y_{v t}}$ is a vector of real valued parameters and denotes the correlation between sentiment $y_{v t}$ and topic distribution; and $Z_{\alpha}$ is a normalization term to ensure that the distribution is normalized so that the sum of the probabilities equals to 1 . Note that not all users have posted tweets at every time such as the user $v_{2}$ at time 2 .

As the discussion above, the sentimental status of a user $v$ at time $t$ will be influenced by the sentimental status of her friends at $t-1$, and here we also integrate the user $v$ 's interest to refine the spreading influence. Then we defined the interest-based spreading factor $\xi\left(y_{v t}, y_{u t-1}, x_{u t-1}, l_{v}\right)$ as

$$
\begin{aligned}
\xi\left(y_{v t}, y_{u t-1}, x_{u t-1}, l_{v}\right)= \\
\frac{1}{Z_{\gamma}} \exp \left\{\gamma_{v u}\left(l_{v}{ }^{T} x_{u t-1}\right) I\left(y_{v t}, y_{u t-1}\right)\right\}
\end{aligned}
$$

where $\gamma_{v u}$ is a real value indicating the link strength between linked user $v$ and $u, l_{v}^{T} x_{u t-1}$ is refined term that denotes the extent that user $v$ is interested in the tweet posted by another user $u$ at time $t-1, I(\cdot)$ is defined as an indicator function and $Z_{\gamma}$ is a normalization term. Here, we assume that all the tweets posted by user $u$ can be read by user $v$ that follows $u$.

We next define self-spreading factor function as

$$
\partial\left(y_{v t}, y_{v t-\Delta t}\right)=\frac{1}{Z_{\beta}} \exp \left\{\beta_{\Delta t, y_{v t}} I\left(y_{v t}, y_{v t-\Delta t}\right)\right\}
$$

where $\beta_{\Delta t, y_{v t}}$ is weight parameter with respective to time interval $\Delta t$ and the sentimental status of user $v$ at time $t$ and $Z_{\beta}$ is a normalization term. In practice, we set the upper bound of $\Delta t$ as 2 unit timestamps $(1 \leq \Delta t \leq 2)$ to reduce the computational complexity of the proposed model.

By integrating all the factor functions together and according to the Hammersley-Clifford theorem (Hammersley and Clifford 1971) we can define the joint distribution over $\mathrm{Y}$ as

$$
\begin{aligned}
& p(Y \mid G) \\
& =\prod_{v=1}^{N} \prod_{t=1}^{T} \ell\left(y_{v t}, x_{v t}\right) \prod_{v=1}^{N} \prod_{t=1}^{T} \prod_{\Delta t=1}^{\Delta T} \partial\left(y_{v t}, y_{v t-\Delta t}\right) \\
& \quad \cdot \prod_{t=1}^{T} \prod_{e_{v u} \in E} \xi\left(y_{v t}, y_{u t-1}, x_{u t-1}, l_{v}\right) .
\end{aligned}
$$

\subsection{Model Learning}

Learning TSSM is to estimate a parameter configuration $\vartheta=(\alpha, \beta, \gamma)$, so that the log-likelihood of observation information (labeled sentimental status of all users from time 1 to time $t-1$ ) are maximized. For presentation simplicity, 



Figure 2: Graphical representation of the TSSM

we concatenate all factor functions. The joint probability defined in (Eq. 5) can be rewritten as

$$
\begin{aligned}
& p(Y \mid G)=\frac{1}{Z} \exp \left\{\sum_{v=1}^{N} \sum_{t}^{T} \alpha_{y_{v t}} x_{v t}\right\} \\
& \times \exp \left\{\sum_{v=1}^{N} \sum_{t=1}^{T} \sum_{\Delta t=1}^{\Delta T} \beta_{\Delta t} \cdot I\left(y_{v t}, y_{v t-\Delta t}\right)\right\} \\
& \times \exp \left\{\sum_{t=1}^{T} \sum_{e_{v u} \in E} \gamma_{v u} \cdot\left(x_{u t-1} \cdot l_{v}\right) \cdot I\left(y_{v t}, y_{u t-1}\right)\right\} \\
& =\frac{1}{Z} \exp \left\{\vartheta^{T} S\right\},
\end{aligned}
$$

where $Z=Z_{\alpha} Z_{\beta} Z_{\gamma}$ is a normalization factor, $S$ is the aggregation of factor functions over all sentimental status nodes.

One challenge for learning the TSSM model is that the input data is partially-labeled. To calculate $Z$, one needs to sum up the likelihood of possible states for all nodes including unlabeled nodes. To deal with this, we use the labeled data to infer the unknown labels. Here $Y \mid Y^{L}$ denotes a labeling configuration $Y$ inferred from the known labels. Thus, we can define the following log-likelihood objective function $O(\vartheta)$ :

$$
\begin{aligned}
\mathrm{O}(\vartheta)= & \log \left(Y^{L} \mid G\right)=\log \sum_{Y \mid Y^{L}} \frac{1}{Z} \exp \left\{\vartheta^{T} S\right\} \\
& =\log \sum_{Y \mid Y^{L}} \exp \left\{\vartheta^{T} S\right\}-\log Z \\
& =\log \sum_{Y \mid Y^{L}} \exp \left\{\vartheta^{T} S\right\}-\log \sum_{Y} \exp \left\{\vartheta^{T} S\right\} .
\end{aligned}
$$

To solve the objective function, we can consider a gradient decent method (or a Newton-Raphson method). Specifically, we first calculate the gradient for each parameter $\vartheta$ :

$$
\begin{array}{r}
\frac{\partial O(\vartheta)}{\partial \vartheta}=\frac{\partial\left(\log \sum_{Y \mid Y_{L}} \exp \left\{\vartheta^{T} S\right\}-\log \sum_{Y} \exp \left\{\vartheta^{T} S\right\}\right)}{\partial \vartheta} \\
=\frac{\sum_{Y \mid Y_{L}} \exp \left\{\vartheta^{T} S\right\} \cdot S}{\sum_{Y \mid Y^{L}} \exp \left\{\vartheta^{T} S\right\}}-\frac{\sum_{Y} \exp \left\{\vartheta^{T} S\right\} \cdot S}{\sum_{Y} \exp \left\{\vartheta^{T} S\right\}} \\
=E_{p_{\vartheta}\left(Y \mid Y^{L}, G\right)} S-E_{p_{\vartheta}(Y \mid G)} S .
\end{array}
$$

Another challenge here is that the graphical structure in TSSM can be arbitrary and may contain cycles, which makes it intractable to directly calculate the second expectation $E_{p_{\vartheta}(Y \mid G)} S$. A number of approximate algorithms have been proposed, such as Loopy Belief Propagation (LBP) (Murphy, Weiss, and Jordan 2013). In this paper, we utilize Loopy Belief Propagation.

We briefly introduce the LBP algorithm here. The main idea of the LBP algorithm is passing messages on the factor graph. There are two types of messages:

(1) A message from the variable node $v$ to the factor node $\partial_{i}$ (or $\left.\xi_{i}\right)$ denoted as:

$$
\mu_{v \rightarrow \partial_{i}}(v)=\prod_{j \in n e(v) \backslash \partial_{i}} \lambda_{\partial_{i} \rightarrow v}(v)
$$

which means the message from $v$ to $\partial_{i}$ is the product of the messages from neighboring factor nodes of $v$ except $\partial_{i}$.

(2) A message from the factor node $\partial_{i}$ to the variable node $v$, denoted as:

$$
\lambda_{\partial_{i} \rightarrow v}(v)=\sum_{u \in n e\left(\partial_{i}\right) \backslash v} \partial_{i} \prod_{u \in n e\left(\partial_{i}\right) \backslash v} \mu_{u \rightarrow \partial_{i}}(u)
$$

which means the message from $\partial_{i}$ to $v$ is the product of the factor $\partial_{i}$ with the messages from all other connecting variable nodes, marginalized over all variable nodes except $v$ (i.e., $n e\left(\partial_{i}\right) \backslash v$ ).

If the variable $v$ has a label $y_{v}$, the message it sends to any factor nodes is 1 if $v=y_{v}$ and 0 otherwise. As the messages are defined recursively, we initialize all messages to the uniform distribution and then update the messages according to Eq. 9 and Eq. 10 iteratively. With the final messages, we can compute the marginal probability of the variable $v$ as:

$$
p(v) \propto \prod_{j \in n e(v)} \lambda_{\ell_{j} \rightarrow v}(v) .
$$

We can also compute the marginal probability of the variable set involved in the factor $\ell_{i}$ as:

$$
p\left(n e\left(\partial_{i}\right)\right) \propto \partial_{i} \prod_{u \in n e\left(\ell_{i}\right)} \mu_{u \rightarrow \ell_{i}}(u) .
$$




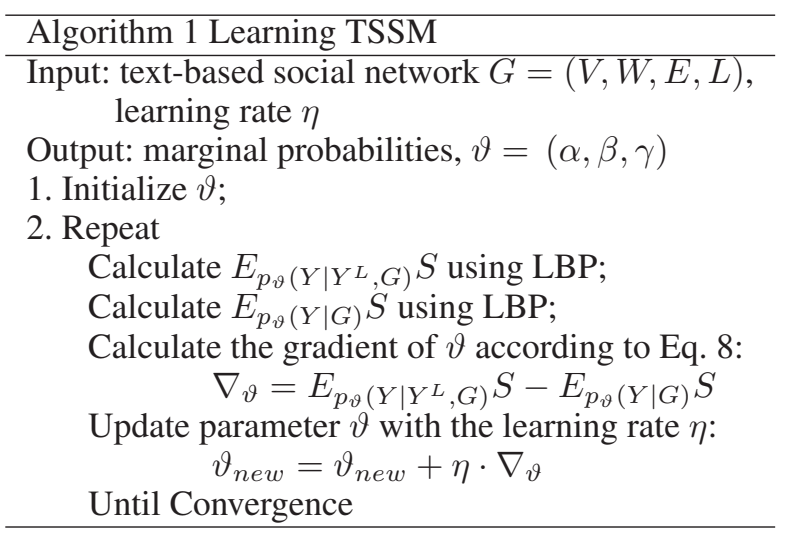

Thus, we approximate marginal probabilities $P\left(y_{v t} \mid \vartheta\right)$, $P\left(y_{v t}, y_{u t-1} \mid \vartheta\right)$ and $P\left(y_{v t}, y_{v t-\Delta t} \mid \vartheta\right)$ using LBP. With the marginal probabilities, the gradient can be obtained by summing over all sentimental status nodes. It is worth noting that we need to perform the LBP process twice in each iteration, one time for estimating the marginal probability and the other for. Finally, with the gradient, we update each parameter with a learning rate $\eta$. The learning algorithm is summarized in Algorithm 1.

Inferring Unknown Sentimental Status. We now turn to describe how to infer the type of unknown sentimental status. Based on learned parameters $\vartheta$, we can predict the label of each sentimental status by finding a label configuration which maximizes the joint probability (Eq. 5), i.e.,

$$
Y^{*}=\arg \max _{Y \mid Y^{L}} P(Y \mid G) \text {. }
$$

That is, we predict the type of a sentimental status as the label with largest marginal probability. The marginal probability is then taken as the prediction confidence.

\subsection{Experimental Setup}

\section{Experiment}

Data Set. We use the public Twitter dataset (Rui et al. 2012) collected in May 2011. It crawled at most 600 tweets for each user from December 2010 to July 2011. As some users' tweets were not available due to users' privacy settings or simply because they did not post many tweets, only 147,909 users' tweets were crawled with their following relationships. Because the time span of the entire data set is long, it does not match the suddenness of sentiment spreading. We use two subsets of the dataset from March 1, 2011 to March 20, 2011 corresponding to the time when the iPad was launched and from May 1, 2011 to May 10, 2011 corresponding to the event of Bin Laden's Death. Respective data is shown in Table 1. In these two datasets, the continuous time is converted into a set of short periods. One of the periods is called a timestamp. In the experiment, to ensure that most users at least have a piece of tweet at each timestamp, we set the time step to two days.

Topic and User Interest Discovery. We applied TwitterLDA model (Zhao et al. 2011) to automatically identify
Table 1: Statistics of datasets

\begin{tabular}{c|c|c|c|c}
\hline Dataset & \#users & \#tweets & \#edges & \#timestamp \\
\hline Twittr3 & 60602 & $1,876,930$ & 567,474 & 10 \\
Twitter5 & 68486 & $1,577,493$ & 789,094 & 5 \\
\hline
\end{tabular}

the topics of every original tweet and user interests in the data set. We first remove all stop words, slang words, and non-English phrases. Next, we iteratively filter away words, tweets, and users such that: each word must appear in at least 3 remaining tweets, each tweet contains at least 3 remaining words, and each user has at least 20 remaining tweets as did in (Hoang and Lim 2016). These minimum thresholds are designed to ensure that for each user, tweet, and word, we have enough observations.

Based on the learnt topics of tweets and topic distributions of users (i.e., users' interests $L$ ) we can compute the topic distribution of tweets posted by user $u$ at time $t$

$$
x_{v t} \propto \sum_{j=1}^{N_{v t}} \theta_{v t, j}
$$

where $N_{v t}$ denotes the number of tweets posted by user $v$ at time $t$ and $\theta_{v t, j}$ denotes the topic distribution of $j$ th tweet posted by user $v$ at time $t$.

Sentimental Status Discovery. How to measure sentimental status of users is a key issue in our method. Note that at each timestamp, one user may post several tweets. Here we used the main sentiment of the tweets as the sentimental status of a user at a timestamp as did in (Xiong et al. 2018).

However, facing the vast scale of tweets, manually labeling is powerless. Instead, a tool called SentiStrength (Thelwall, Buckley, and Paltoglou 2012; Thelwall and Buckley 2013) is utilized in our work to annotate tweets with positive and negative sentiment scores. By comparing with other tools, SentiStrength provides several advantages: it is designed for short informal texts with abbreviations and slang (features commonly observed in Twitter), and it employs linguistic rules for negations, amplifications, booster words, emotions, spelling corrections, which are particularly wellsuited to process social media data.

By applying SentiStrength, each tweet is assigned a positive $R^{+}(w)$ and negative $R^{-}(w)$ sentimental score. Both scores are on a scale ranging between 1 (neutral) and 5 (strongly positive or negative). To capture the sentiment expressed by each tweet in one single measure, we define the polarity score $R(w)$ of tweet $w$ as the sum of positive and negative sentimental scores in the following equation:

$$
R(w)=R^{+}(w)-R^{-}(w)
$$

The polarity score $R(w)$ ranges between -4 (extremely negative, when $R^{+}(w)=1$ and $R^{-}(w)=5$ ) to +4 (extremely positive, when $R^{+}(w)=5$ and $\left.R^{-}(w)=1\right)$. When the positive and negative sentimental scores for a tweet are the same $\left(R^{+}(w)=R^{-}(w)\right)$, we say that the polarity of the tweet is neutral (i.e. $R(w)=0$ ). When $R(w)<0$ or $R(w)>$ 0 , we say that the polarity of the tweet is negative or positive respectively as did in (Xiong et al. 2018). 
Table 2: Performance of sentimental status inference

\begin{tabular}{|c|c|c|c|c|c|c|c|}
\hline \multirow{2}{*}{ Class } & \multirow{2}{*}{ Method } & \multicolumn{3}{|c|}{ Twitter3 } & \multicolumn{3}{|c|}{ Twitter5 } \\
\hline & & Precision & Recall & F1-score & Precision & Recall & F1-score \\
\hline \multirow{5}{*}{ Negative } & LibSVM & 0.1811 & 0.0789 & 0.1099 & 0.3600 & 0.0070 & 0.0137 \\
\hline & $\mathrm{BN}$ & 0.3801 & 0.1011 & 0.1600 & 0.3590 & 0.0600 & 0.1030 \\
\hline & LR & 0.4820 & 0.0711 & 0.1220 & 0.4130 & 0.0360 & 0.0650 \\
\hline & EIC & 0.2684 & 0.1451 & 0.1883 & 0.2140 & 0.1179 & 0.1520 \\
\hline & TSSM & 0.5981 & 0.3502 & 0.4417 & 0.5734 & 0.2892 & 0.3844 \\
\hline \multirow{5}{*}{ Neutral } & LibSVM & 0.8250 & 0.9641 & 0.8891 & 0.7036 & 0.8716 & 0.7786 \\
\hline & $\mathrm{BN}$ & 0.8430 & 0.9290 & 0.8840 & 0.7240 & 0.8230 & 0.7710 \\
\hline & LR & 0.8350 & 0.9740 & 0.8990 & 0.7170 & 0.8510 & 0.8180 \\
\hline & EIC & 0.8010 & 0.6386 & 0.7106 & 0.6894 & 0.5221 & 0.6236 \\
\hline & TSSM & 0.9101 & 0.9671 & 0.9377 & 0.8595 & 0.9658 & 0.9095 \\
\hline \multirow{5}{*}{ Positive } & LibSVM & 0.4011 & 0.1090 & 0.1714 & 0.412 & 0.1652 & 0.2358 \\
\hline & $\mathrm{BN}$ & 0.3850 & 0.2880 & 0.3300 & 0.3860 & 0.3790 & 0.3830 \\
\hline & LR & 0.5730 & 0.2310 & 0.3289 & 0.4810 & 0.2390 & 0.3380 \\
\hline & EIC & 0.2322 & 0.4324 & 0.3021 & 0.3367 & 0.5051 & 0.4040 \\
\hline & TSSM & 0.6600 & 0.5521 & 0.6012 & 0.6209 & 0.4555 & 0.5254 \\
\hline
\end{tabular}

Task and Evaluation Metrics. After above preprocessing for the data sets, we can obtain the input network G, users' interests $L$, topic distributions of tweets $X$ and emotion contagion history $Y$, we construct a training dataset $\left\{\left(x_{v t}, y_{v t}\right)\right\}_{v \in V, t=1 \ldots T}$, where $x_{v t}$ is the topic distribution posted by user $v$ at time $t$, and $y_{v t}$ indicated the user $v$ 's sentimental status at time $t$. The task in our experiment is to predict users' sentimental status in future (time $t+1$ ). For evaluation, we consider the following performance metrics: Precision, Recall, and F1-score.

Comparison Methods. Since we have a training dataset and predict users' sentimental status at time $t+1$ (i.e., test dataset), we choose some classical classification methods, which ignore the sentimental influence between users, to indicate the significance of studying sentiment spreading.

SVM: it uses all topic distributions as features associated with each user to train a classification model, and then applies it to predict users' sentimental status in test data. For SVM, we use LibSVM (Chang and Lin 2011).

LR and NB: we also use logistic regression and Naive Bayes to train the classification model with the same features as those in the SVM method. For these methods, we employ Weka (Frank, Hall, and Witten 2016).

In addition, we also choose a recent sentiment spreading method which uses information of network and retweeting behavior to define the influence strength between linked users, to prove the significance of our method.

EIC: we also compared with EIC (Wang et al. 2017), an emotion-based independent cascade model for sentiment spreading. The idea of EIC is that some active users are selected to spread tweets, which contain sentiments, to inactive users in the network. Thus, we here treat the users from time 1 and $t$ as active users and all users at time $t+1$ as inactive user. The EIC model categorizes sentiment into fine-grained classes. Then we classified sentiment in the EIC model into three types. Happiness was viewed as positive; surprise was viewed as neutral; anger, sadness, fear and disgust were all regarded as negative as did in (Xiong et al. 2018). Finally we added the values of all kinds of sentiment in an individual's tweets to obtain his/her sentiments at time $t+1$.

The difference between this method and our model is that EIC uses information of retweet behavior to define the influence strength between linked users, while our method integrates topic distributions of tweets into the unified sentiment spreading model to automatically learn the influence strength between users and refine the influence strength by user interest.

\subsection{Results and Analyses}

Prediction Performance. Table 2 lists the results of our model and compared methods. We can see that TSSM achieves the best performance than other out of all considered methods. The results can be summarized as follows.

SVM, LR and NB have no ability to handle the correlation features (i.e., self-spreading and interest-based spreading factor), which hurts negatively impacts the performance. Although EIC considered the influence between users, it ignored the evolution of users' sentimental status over time. More importantly, EIC just used the information of network topologies or user behaviors to define the influence strength between linked users and ignored the correlation between users' sentimental status and topic distributions embedded in tweets. Moreover, our method can automatically learn the spreading strength between each pair of neighbors to relieve the mismatch in the above correlation.; and finally, TSSM integrates user interest into the model to refine the spreading strength. In general, our method is more realistic and makes the sentiment prediction more accurate and effective.

Factor Contribution Analysis. In the proposed model, we define three types of factors that influence the sentiment and its spread: topic factor, self-spreading factor and interest-based spreading factor. Here we show whether these factors contribute as we regard in the prediction task. Specifically, we first use the self-spreading factor alone to train a 
Table 3: Profile of example predicted results of two users' sentimental status at time 5 (Twitter5 dataset). The value after the class represents the probability that the user's sentimental status belongs to this class.

\begin{tabular}{c|c|c|c|c|c|c|c}
\hline \multirow{2}{*}{ User Id } & \multirow{2}{*}{ Num. of Followings } & \multirow{2}{*}{ Num. of Tweets at Time 5 } & \multirow{2}{*}{ Label } & \multirow{2}{*}{ EIC } & \multirow{2}{*}{ LR } & \multicolumn{2}{|c}{ TSSM } \\
\cline { 4 - 8 } & & & & & $\ell$ & $\xi$ \\
\hline 334503 & 15 & 0 & Pos. & Neu. : 0.93 & Pos. & Pos.: 0.76 & Pos. : 0.79 \\
\hline 15537895 & 23 & 0 & Neu. & Neu. : 0.65 & - & - & Neu. : 0.98 \\
\hline
\end{tabular}

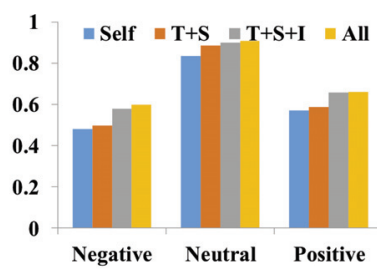

(a)

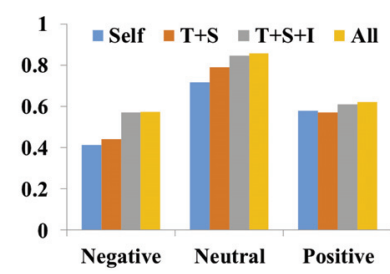

(b)
Figure 3: Factor analysis. The performance (Precision) on (a) Twitter3 and (b) Twitter5.

model (referred to as Self) as a basis model, which has been proven to exist (Cai et al. 2018). Then we incrementally add the topic factor (referred to as $\mathrm{T}+\mathrm{S}$ ), interest-based spreading factor excluding the user interest refined term (referred to as $\mathrm{T}+\mathrm{S}+\mathrm{I}$ ) and interest-based spreading factor including the user interest refined term (referred to as All) as the stepby-step idea we consider, and evaluate their improvements in prediction performance over that using only self-spreading factor. Fig. 3 shows the results. We see that different factors contribute differently to different sentiments. For example, the topic factor is very useful when predicting neutral sentiment. Intuitively, people are neural most of the time, leading to the factor that most tweets are neural ones, and the model has more neutral training data. On the other hand, the interest-based spreading factor improves the performance most for predicting negative sentiment, that because negative sentiments are more likely to infect others and then to spread. Finally, considering users' interests does have the effect of refining the accuracy of the final results.

Effect of User Interest. We study how different extent that a user is interested in the content posted by his friends affect the infection probability. Specifically, we set the extent that a user $v$ at time $t+1$ is interested in the content posted by his friends at time $t$ in Eq. 3 as $x$-axis. And $y$-axis represents the probability that $v$ 's sentiment at time $t+1$ equals to $u$ 's sentiment at time $t$. As Fig. 4 shows, when the extent that a user is interested in the content posted by her friends being negative increases, the probability that she will also be negative grows. However, we also found positive and negative sentiment is more relevant to user interest than neutral ones.

Case Study. We finally use a case study to illustrate the differences between TSSM and the traditional sentiment spreading methods, i.e., EIC. Table 3 shows the profiles of two users and predicted sentimental statuses of these two

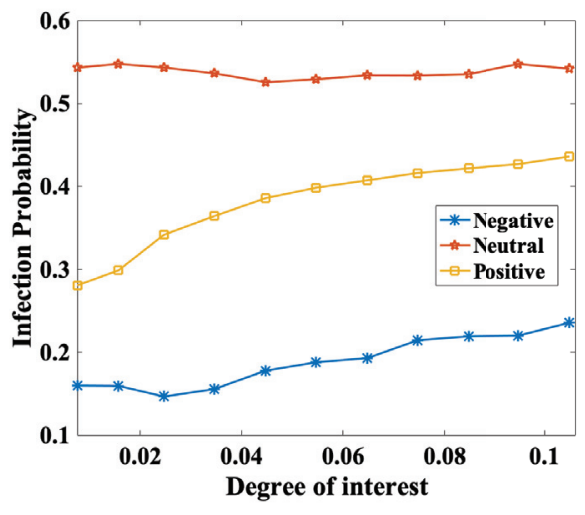

Figure 4: Sentiment spreading analysis on Twitter5. In the figure, $x$-axis indicates the extent that user $v$ is interested in the content posted by his friend $u$, while $y$-axis represents the probability that $v$ 's sentiment at time $t+1$ equals to $u$ 's sentiment at time $t$.

users at time slice 5 on the Twitter5 dataset. To avoid the disturbance of the self-spreading factor, the sentimental statuses of these two users we chose at time slice 5 are different from those at time slice 3 and 4 (since $1 \leq \Delta t \leq 2$ ). For the user jajah, the predicted results of EIC (only using retweet behaviors) and LR (only using the feature of topic distributions) are completely diverse, which indicates that there does exist a disparity between the information of network topologies and tweets. For all that, our method automatically balanced the effect of these two information and learned the interest-based spreading factor (i.e., influence strength) and the topic factor (i.e. correlation between topic distribution embedded in tweet and user sentimental status), both of which suggest the correct predicted sentimental status of the user jajah at time slice 5. In addition, for the example user DigitalSummer who has not post tweets at time slice 5, our method also learned the more explicit result (i.e., 0.98) than the result of EIC (i.e., 0.65), which indicates the essentiality of using user interest to refine the influence strength.

\section{Conclusion}

In this paper, we study the sentiment spreading in text-based social networks. We have proposed a unified probabilistic model TSSM to automatically learn the spreading strength between each pair of neighbors integrating the correlation between topic distributions embedded in tweets and user sentimental status, while we have considered user interest into the model to refine the spreading strength to get more accurate result. We devised an approximate learning algo- 
rithm based on Loopy Belief Propagation to estimate the model parameters. The experiment results validate its superiority compared with the state-of-art methods. As a part of our future work, we plan to extend our TSSM to consider temporal changes of sentimental tendencies for different topics, and apply TSSM to new applications.

\section{Acknowledgments}

The work was supported by the National Key R\&D Program of China (No. 2018YFB1305200), National Natural Science Foundation of China (No. 61772361, 61876128), and the Tianjin Municipal Science and Technology Project (No. 18ZXZNGX00330).

\section{References}

Ahuja, A.; Wei, W.; and Carley, K. M. 2017. Microblog sentiment topic model. In IEEE International Conference on Data Mining Workshops.

Cai, W.; Jia, J.; and Han, W. 2018. Inferring emotions from image social networks using group-based factor graph model. In 2018 IEEE International Conference on Multimedia and Expo (ICME), 1-6. IEEE.

Chang, C.-C., and Lin, C.-J. 2011. Libsvm: A library for support vector machines. ACM transactions on intelligent systems and technology (TIST) 2(3):27.

Frank, E.; Hall, M.; and Witten, I. 2016. The weka workbench (online appendix). data mining.

Guillory, J.; Spiegel, J.; Drislane, M.; Weiss, B.; Donner, W.; and Hancock, J. 2011. Upset now?: emotion contagion in distributed groups. In Proceedings of the SIGCHI conference on human factors in computing systems, 745-748. ACM.

Hao, Z.; Huang, C.; Cai, R.; Wen, W.; Huang, Y.; and Chen, B. 2016. User interest related information diffusion pattern mining in microblog. Pattern Recognition and Artificial Intelligence 29(10):924-935.

He, D.; Feng, Z.; Jin, D.; Wang, X.; and Zhang, W. 2017. Joint identification of network communities and semantics via integrative modeling of network topologies and node contents. In Thirty-First AAAI Conference on Artificial Intelligence.

Hoang, T. A., and Lim, E. P. 2016. Microblogging content propagation modeling using topic-specific behavioral factors. IEEE Transactions on Knowledge and Data Engineering 28(9):2407-2422.

Jin, D.; Wang, X.; He, R.; He, D.; Dang, J.; and Zhang, W. 2018. Robust detection of link communities in large social networks by exploiting link semantics. In Thirty-Second AAAI Conference on Artificial Intelligence.

Kramer, A. D.; Guillory, J. E.; and Hancock, J. T. 2014. Experimental evidence of massive-scale emotional contagion through social networks. Proceedings of the National Academy of Sciences 111(24):8788-8790.

Kschischang, F. R.; Frey, B. J.; Loeliger, H.-A.; et al. 2001. Factor graphs and the sum-product algorithm. IEEE Transactions on information theory 47(2):498-519.
Lagnier, C.; Denoyer, L.; Gaussier, E.; and Gallinari, P. 2013. Predicting information diffusion in social networks using content and user's profiles. In European Conference on Advances in Information Retrieval.

Murphy, K.; Weiss, Y.; and Jordan, M. I. 2013. Loopy belief propagation for approximate inference: An empirical study. In Fifteenth Conference on Uncertainty in Artificial Intelligence.

Rui, L.; Wang, S.; Deng, H.; Rui, W.; and Chang, C. C. 2012. Towards social user profiling: unified and discriminative influence model for inferring home locations. In Acm Knowledge Discovery and Data Mining.

Thelwall, M., and Buckley, K. 2013. Topic-based sentiment analysis for the social web: The role of mood and issuerelated words. Journal of the American Society for Information Science and Technology 64(8):1608-1617.

Thelwall, M.; Buckley, K.; and Paltoglou, G. 2012. Sentiment strength detection for the social web. Journal of the American Society for Information Science and Technology 63(1):163-173.

Varshney, D.; Kumar, S.; and Gupta, V. 2014. Modeling information diffusion in social networks using latent topic information. In International Conference on Intelligent Computing.

Wang, S.; Hu, X.; Yu, P. S.; and Li, Z. 2014. Mmrate: inferring multi-aspect diffusion networks with multi-pattern cascades. In Proceedings of the 20th ACM SIGKDD international conference on Knowledge discovery and data mining, 1246-1255. ACM.

Wang, S.; Yan, Z.; Hu, X.; Philip, S. Y.; and Li, Z. 2015. Burst time prediction in cascades. In Twenty-Ninth AAAI Conference on Artificial Intelligence.

Wang, Q.; Jin, Y.; Yang, T.; and Cheng, S. 2017. An emotion-based independent cascade model for sentiment spreading. Knowledge-Based Systems 116:86-93.

Wu, B.; Jia, J.; Yang, Y.; Zhao, P.; Jie, T.; and Qi, T. 2017. Inferring emotional tags from social images with user demographics. IEEE Transactions on Multimedia PP(99):1-1.

Xiong, X.; Li, Y.; Qiao, S.; Han, N.; Wu, Y.; Peng, J.; and Li, B. 2018. An emotional contagion model for heterogeneous social media with multiple behaviors. Physica A: Statistical Mechanics and its Applications 490:185-202.

Yang, Y.; Jia, J.; Wu, B.; and Tang, J. 2016. Social roleaware emotion contagion in image social networks. In Thirtieth AAAI Conference on Artificial Intelligence.

Zhao, W. X.; Jiang, J.; Weng, J.; He, J.; Lim, E. P.; Yan, H.; and Li, X. 2011. Comparing twitter and traditional media using topic models. In European Conference on Advances in Information Retrieval.

Zhao, L.; Wang, J.; Huang, R.; Cui, H.; Qiu, X.; and Wang, X. 2014. Sentiment contagion in complex networks. Physica A: Statistical Mechanics and its Applications 394:17-23.

Zou, X.; Yang, J.; and Zhang, J. 2018. Microblog sentiment analysis using social and topic context:. Plos One 13(2):e0191163. 